\title{
Reliability-Centered Maintenance Methodology and Application: A Case Study
}

\author{
Islam H. Afefy \\ Industrial Engineering Department, Faculty of Engineering, Fayoum University, Al Fayyum, Egypt \\ E-mail: Islamhelaly@yahoo.com \\ Received September 15, 2010; revised September 27, 2010; accepted October 19, 2010
}

\begin{abstract}
This paper describes the application of reliability-centered maintenance methodology to the development of maintenance plan for a steam-process plant. The main objective of reliability-centered maintenance is the cost-effective maintenance of the plant components inherent reliability value. The process-steam plant consists of fire-tube boiler, steam distribution, dryer, feed-water pump and process heater. Within this context, a maintenance program for the plant is carried out based on this reliability-centered maintenance concept. Applying of the reliability-centered maintenance methodology showed that the main time between failures for the plant equipments and the probability of sudden equipment failures are decreased. The proposed labor program is carried out. The results show that the labor cost decreases from 295200 \$/year to 220800 \$/year (about $25.8 \%$ of the total labor cost) for the proposed preventive maintenance planning. Moreover, the downtime cost of the plant components is investigated. The proposed PM planning results indicate a saving of about $80 \%$ of the total downtime cost as compared with that of current maintenance. In addition, the proposed spare parts programs for the plant components are generated. The results show that about $22.17 \%$ of the annual spare parts cost are saved when proposed preventive maintenance planning other current maintenance once. Based on these results, the application of the predictive maintenance should be applied.
\end{abstract}

Keywords: Reliability, RCM, Preventive Maintenance, FMEA, Case Study

\section{Introduction}

Steam system is an important part of many processing. Maintenance, availability, reliability and total maintenance reliability cost are some of the most important factors of steam-process plant. The plant provides heat energy to Egyptian Minerals and Salts Company (EMISAL), EL-Fayoum, Egypt. The main product of the company is sodium sulphate unhydrous and sodium chloride.

This work aims to generate a maintenance program that based on the RCM technique for the process-steam plant components. This technique should be able to minimize the downtime (DT) and improve the availability of the plant components. Also, it should benefits to decrease the spare parts consumption system components.

RCM is a systematic approach to determine the maintenance requirements of plant and equipment in its operating [1]. It is used to optimize preventive maintenance (PM) strategies. The developed PM programs minimize equipment failures and provide industrial plants with effective equipment [2]. RCM is one of the best known and most used devices to preserve the operational efficiency of the steam system. RCM operates by balancing the high corrective maintenance costs with the cost of programmed (preventive or predictive) polices, taking into account the potential shortening of "useful life" of the item considered. But it is difficult to select suitable maintenance strategy for each piece of equipment and each failure mode, for the great quantity of equipment and uncertain factors of maintenance strategy decision $[3,4]$.

RCM philosophy employs preventive maintenance, predictive maintenance (PdM), real-time monitoring (RTM), run-to-failure (RTF) and proactive maintenance techniques is an integrated manner to increase the probability that a machine or component will function in the required manner over its design life cycle with a minimum of maintenance $[5,6]$. 


\section{Reliability-Centered Maintenance Methodology}

Reliability-centered maintenance (RCM) is the optimum mix of reactive, time or interval-based, condition-based, and proactive maintenance practices. These principal maintenance strategies, rather than being applied independently, are integrated to take advantage of their respective strengths in order to maximize facility and equipment reliability while minimizing life-cycle costs.

Total productive maintenance (TPM), total maintenance assurance, preventive maintenance, reliabilitycentered maintenance (RCM), and many other innovative approaches to maintenance problems all aim at enhancing the effectiveness of machines to ultimately improve productivity [7].

\subsection{Reliability-Centered Maintenance Components}

The components of RCM program are shown in Figure 1. This figure showing that RCM program consists of (reactive maintenance, preventive maintenance, condition based maintenance, and proactive maintenance) and its patterns.

As shown in Figure 2, the RCM steps are presented. The steps describe the systematic approach used to implement the preserves the system function, identifies failure mode, priorities failure used to implement the preserves the system function, identifies failure mode, priorities failure modes and performs PM tasks. The RCM steps are as follows [8]:

- Step1: system selection and data collection.

- $\quad$ Step2: system boundary definition.

- $\quad$ Step3: system description and functional block.

- $\quad$ Step4: system function functional failures.

- $\quad$ Step5 : failure mode effect analysis

- Step6: logic tree diagram.

- $\quad$ Step7: task selection.

\subsection{System Selection and Data Collection}

Determining the list of the system components is one of the first steps in RCM. The criticality analysis requires different kind of data of each component that build up the system. The effect of failure of the system main components may effect system productivity and maintenance cost. The factors effecting selection of critical system are as follows:

1) Mean-time between failures (MTBF).

2) Total maintenance cost.

3) Mean time to repair (MTTR).

4) Availability.

\subsection{Logic Tree Analysis (LTA)}

The basic (LTA) uses the decision tree structure shown in Figure 3. From this figure, decision bins: 1) safetyrelated, 2) outage-related, or 3) economic-related were noticed. Each failure mode is entered into the top box of the tree, where the first question is posed: Does the operator, in the normal course of his or her duties, know that something of an abnormal or detrimental nature has occurred in the plant? It is not necessary that the operator know exactly what is awry for the answer to be yes [6].

\subsection{Criticality Analysis}

Criticality analysis is a tool used to evaluate how equipment failures impact organizational performance in order to systematically rank plant assets for the purpose of work prioritization, material classification, PM/PdM development and reliability improvement initiatives [9]. In general, failure modes, effects and criticality analysis (FMEA/FMECA) requires the identification of the following basic information in Table 1. In Figure 4, algorithm for the calculation of equipments criticality is presented. This figure shows the calculation steps of the equipments criticality

The criticality is assessed based on the effect of er-

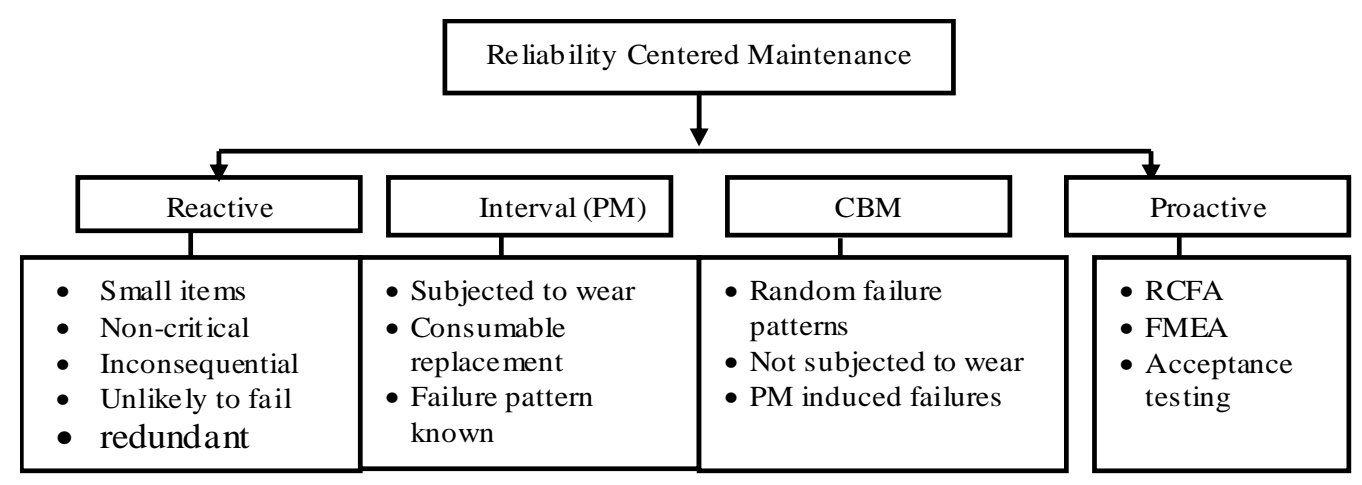

Figure 1. Components of RCM program. 


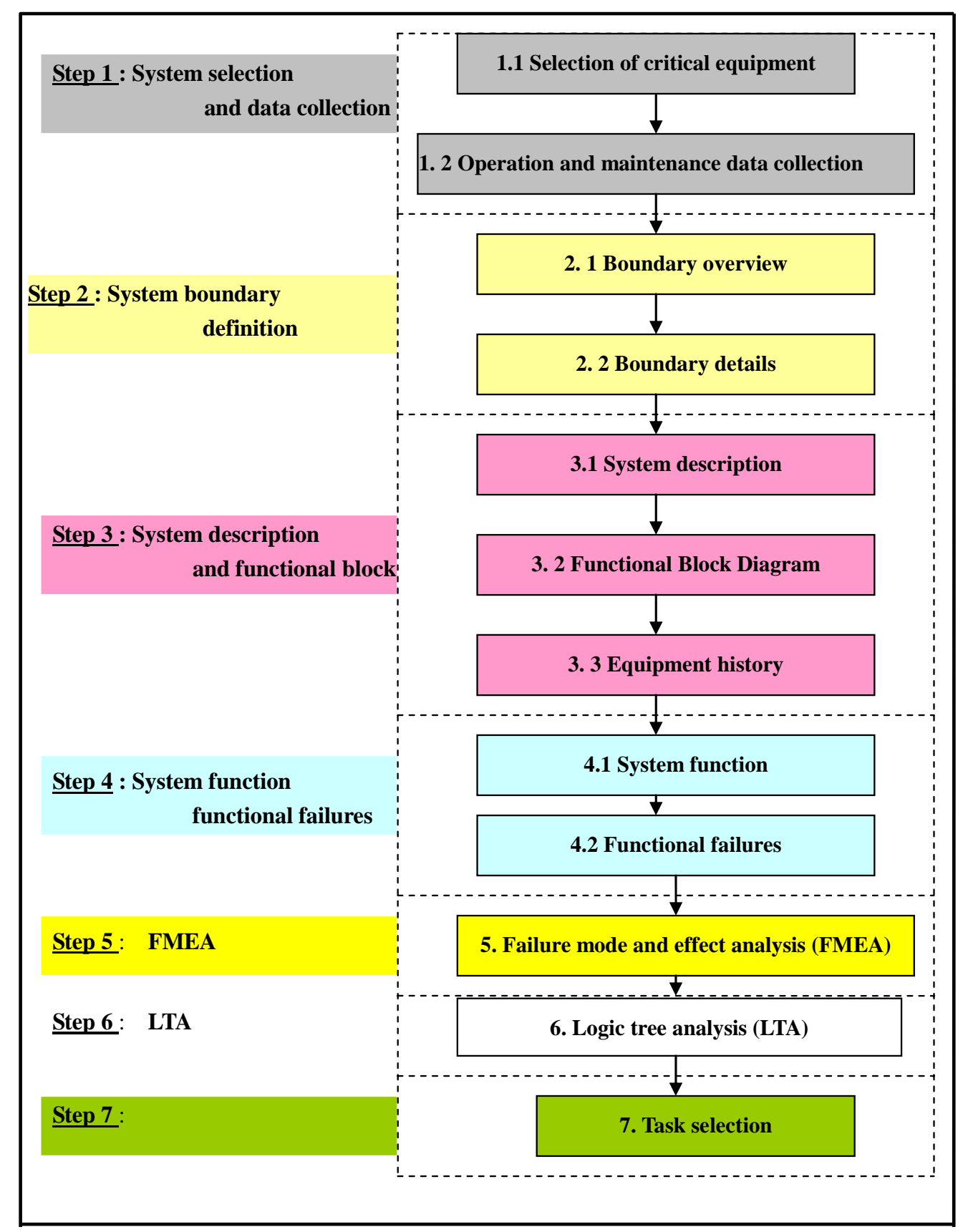

Figure 2. Main steps of the RCM.

rors/faults and on the time from the occurrence until the effect occurs on the installation and is quantified with 1,2 , 3 in Table 1.

where,

$$
\mathrm{EC}=(30 * \mathrm{P}+30 * \mathrm{~S}+25 * \mathrm{~A}+15 * \mathrm{~V}) / 3
$$

EC: is the equipment criticality, \%

$\mathrm{P}$ : is the product

$\mathrm{S}$ : is the safety

$\mathrm{A}:$ is the equipment stand by

$\mathrm{V}$ : is the capital cost.

\section{Case Study}

We select the most critical system in the sodium chloride plant which contains the most critical items. The plant provides heat energy to perform the drying process for the sodium chloride anhydrous lead to the aimed degree.

\subsection{System Description}

The structure of the steam-process plant is presented in 


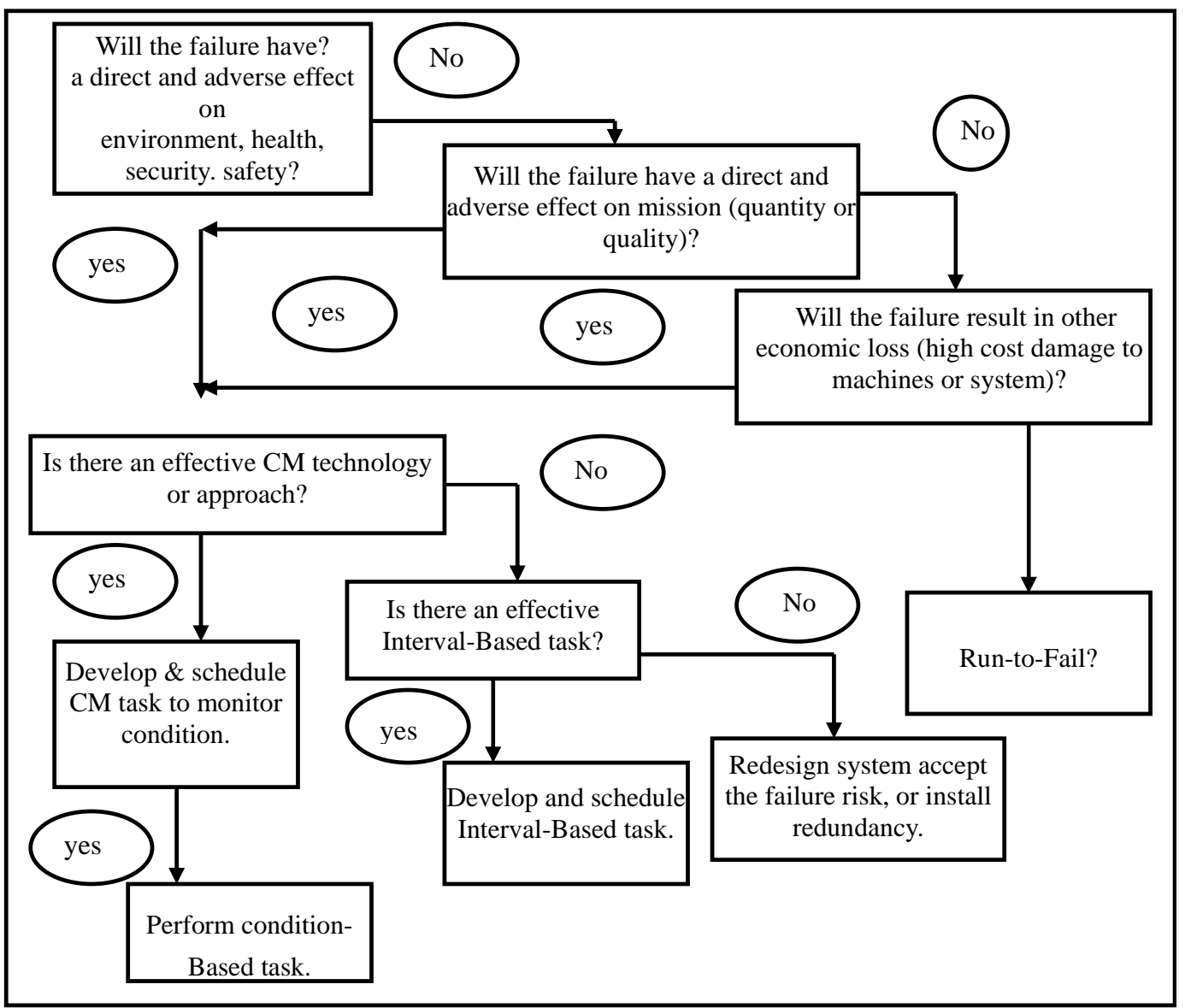

Figure 3. Reliability-centered maintenance (RCM) logic tree.

Table 1. Criticality analysis.

\begin{tabular}{|c|c|c|c|}
\hline Criteria & & Weight & Levels \\
\hline Impact on production & $\mathrm{P}$ & $30 \%$ & $\begin{array}{r}\text { (3) Very important } \\
\text { (2) Important } \\
\text { (1) normal }\end{array}$ \\
\hline Impact on safety & $\mathrm{S}$ & $30 \%$ & $\begin{array}{r}\text { (3)Very important } \\
\text { (2) Important } \\
\text { (1) normal }\end{array}$ \\
\hline Availability of standby & A & $25 \%$ & $\begin{array}{l}\text { (3) Without standby } \\
\text { (2) With stand by and medium availability, and } \\
\text { (1) With standby and high availability }\end{array}$ \\
\hline Equipment value & $\mathrm{V}$ & $15 \%$ & $\begin{array}{l}\text { (3)High value } \\
\text { (2) normal, and } \\
\text { (1) Low value }\end{array}$ \\
\hline
\end{tabular}

Figure 5. The steam-process plant consists of a fire-tube boiler, feed-water pump, condensate tank, dryers and heat exchanger $(\mathrm{PH})$.

Fire-tube boiler components are shown in Figure 6. A fire-tube boiler is a type of boilers in which hot gases from the fire pass through one or more tubes within the boiler. It is one of the two major types of boilers, the other being the water tube boiler. A fire tube boiler can be either horizontal or vertical. A fire-tube boiler is sometimes called a "smoke-tube" or "shell boiler" boiler.

\subsection{System Boundary Definition}

Some gross system definition and boundaries have been established in the normal course of the plant or facility design, and these system definitions have already been used as the basis of system selection. These same definitions serve quite well to initially define the precise boundaries that must be identified for the RCM analysis process [9]. 


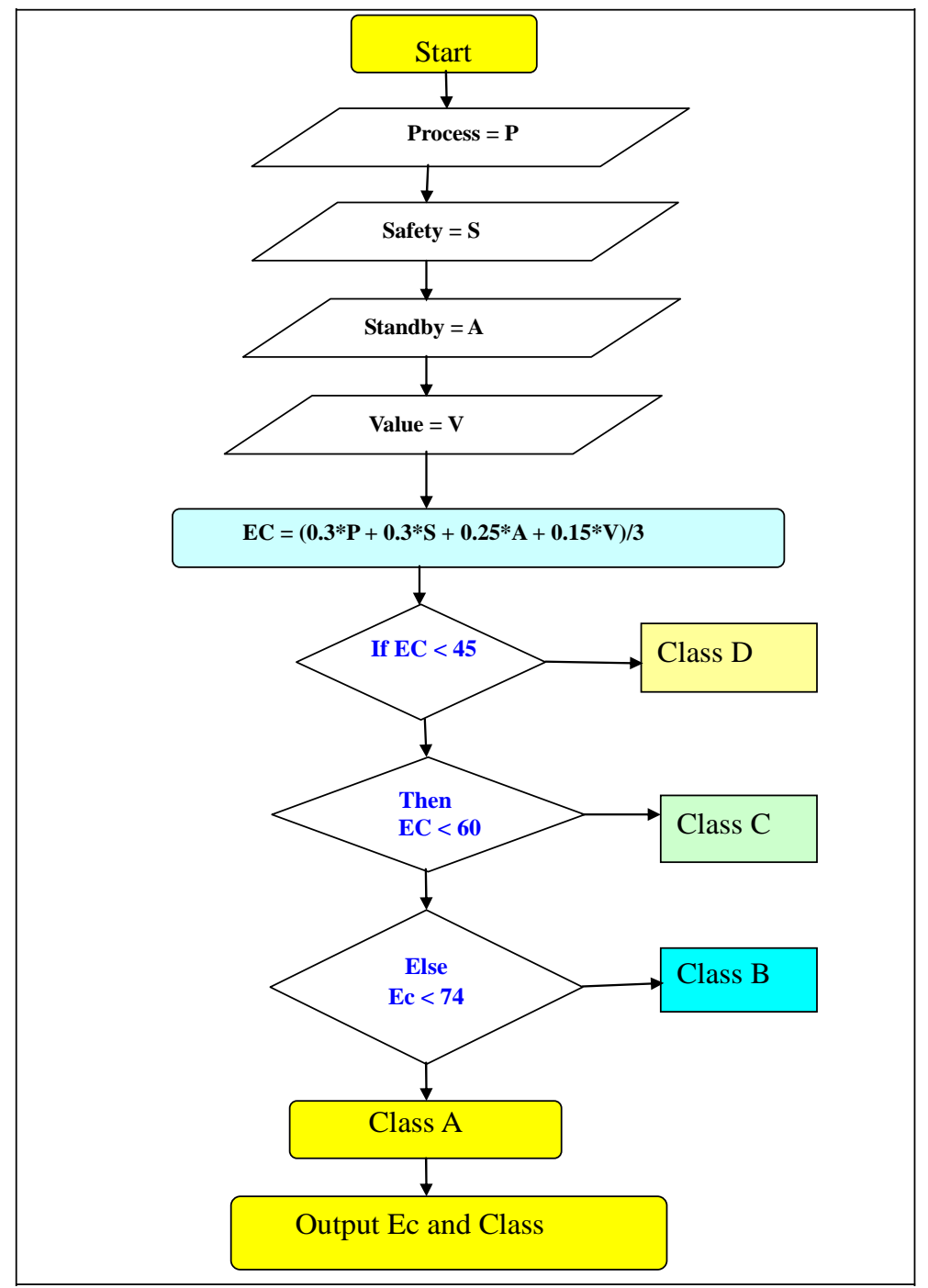

Figure 4. Algorithm for the calculation of equipments criticality.

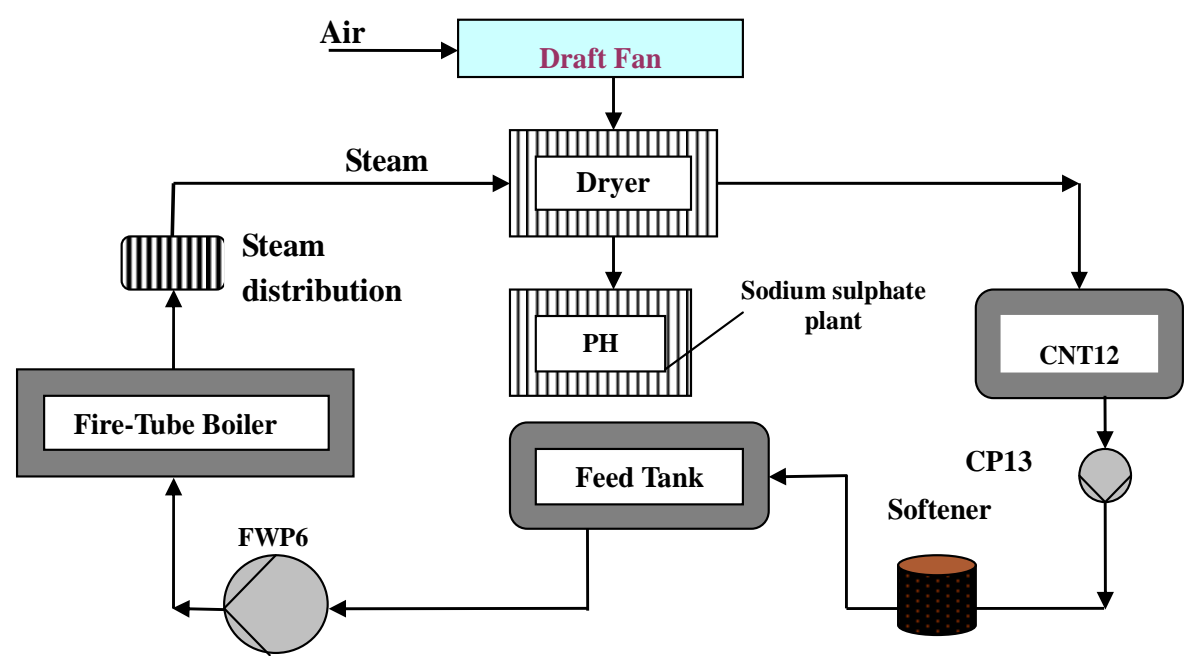

Figure 5. The structure of steam-process plant. 


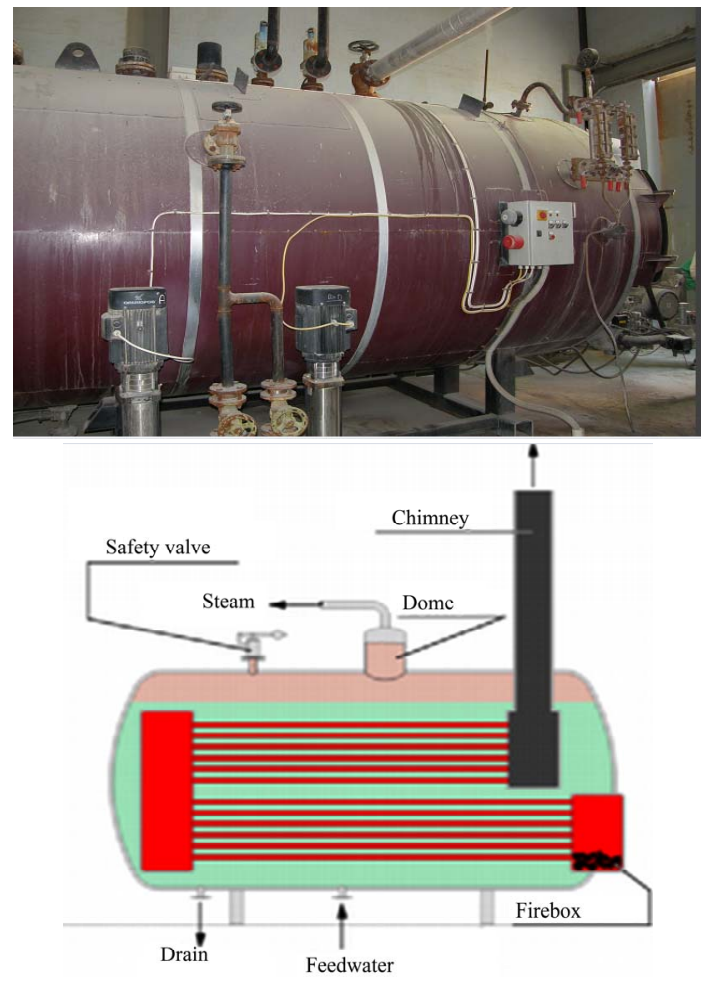

Figure 6. Fire-tube boiler.

\subsubsection{Boundary Overview (see Table 2)}

Table 2. Boundary overview.

\begin{tabular}{l}
\hline 1.Major facilities included: \\
- $\quad$ Concentration Ponds. \\
- $\quad$ Sodium Sulphate. \\
- $\quad$ Sodium Chloride Plant. \\
- $\quad$ Central Laboratories. \\
\hline 2. primary Physical bondries: \\
- $\quad$ Brine entrance to the concentration ponds. \\
- $\quad$ Brine exit from the concentration ponds. \\
- $\quad$ Steam entrance to the sodium sulphate plant. \\
- $\quad$ Ac electric power entrance to the sodium chloride plant \\
- $\quad$ Remain brine entrance to the sodium sulphate plant. \\
- $\quad$ Sodium chloride, as a product, exit from the sodium \\
sulphate plant. \\
- $\quad$ Remain brine exit from the sodium chloride plant. \\
\hline
\end{tabular}

\subsection{Information Collection}

Uniformity, by researching some of the necessary system documents and information that will be needed in subsequent steps, the absence of documentation and data records was a huge problem that makes the system analysis process more difficult. Thus, a greater effort must be done to collect the missing data.

1) Some cards that contain few maintenance actions that have been under taken to some equipments.
2) Some of the operating and maintenance manuals for a few number of equipment.

All of the other information has been collected through a walk down through EMISAL faculties, and personal meeting with EMISAL staff. Fortunately, in most situations, there are plant personnel on site who have essential elements of this data stored either in their desks or their minds. Also Original Equipment Manufacturer (OEM) recommendation stands ready to supply some information.

\subsection{Functional Block Diagram}

The Functional block diagram for the process is introduced in Figure 7. This figure shows the input resource and output for the system main components.

\subsection{System Root Cause Failure Analysis (RCFA)}

As shown in Tables 3 and $\mathbf{4}$, root cause failure analysis for critical equipments in steam system (fire tube boiler and feeding pump) is presented. The cause analysis (failure mode, reason and root cause) for the most critical equipments in the steam system which is [9]:

1) Fire tube boiler.

2) Multi-stage centrifugal pump.

\subsection{Failure Mode and Effect Analysis (FMEA)}

Failure mode and effect analysis is a tool that examines potential product or process failures, evaluates risk priorities, and helps determine remedial actions to avoid identified problems. The spreadsheet format allows easy review of the analysis. Failure mode and effect analysis help on identifying and the creation of functional failure.

At the following tables (Tables 5 and 6) we will represent the failure Mode and effect analysis for the same two equipments we represent its root cause failure analysis [9]:

1) Fire-tube boiler.

2) Multi-stage centrifugal pump.

\subsection{Criticality Analysis for Plant Components}

Then the safety related effects take weight of $40 \%$, Production related effects $40 \%$, and the cost related effects $20 \%$. The failure mode categories A, B, C, and D depending on the criticality index are as shown in Table 7. Tables 8 and 9 show the criticality analysis for boiler, pump, respectively.

\subsection{Task Selection}

A great strength of RCM is the way it provides simple, precise and easily understood criteria for deciding which 


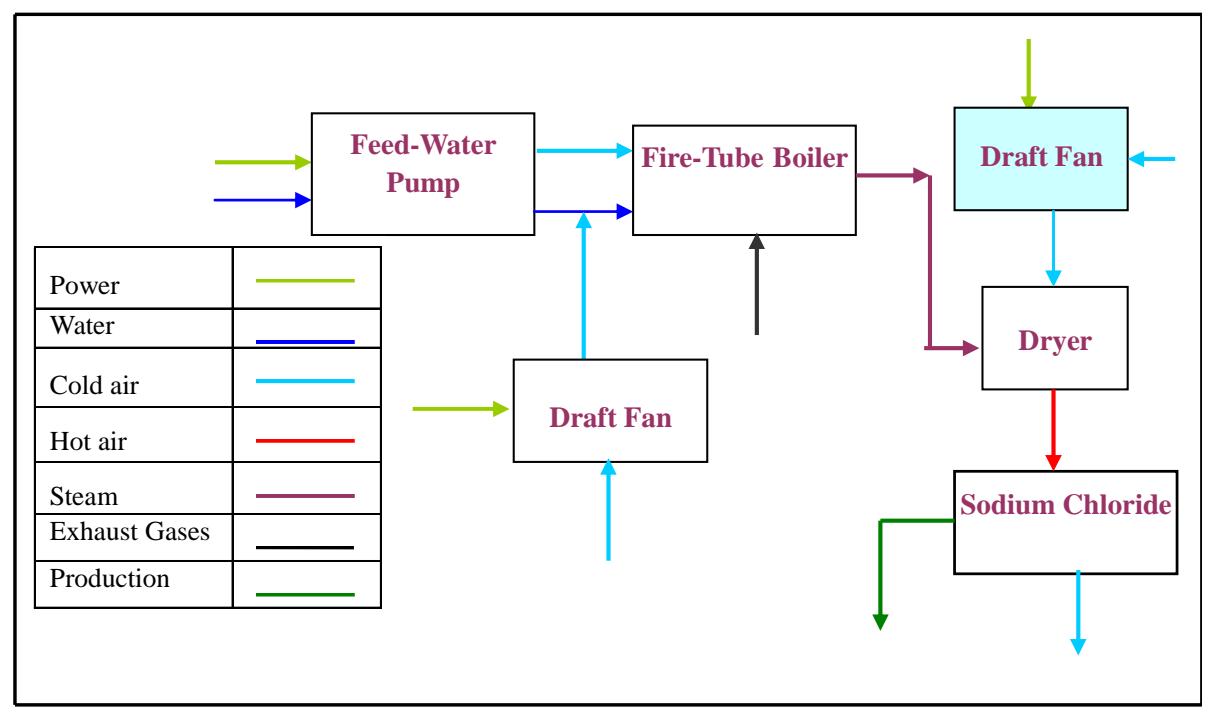

Figure 7. The functional block diagram.

Table 3. Boiler Root Cause Failure Analysis.

\begin{tabular}{|c|c|c|c|}
\hline Failure Mode & Mechanism & Reason & Root Cause \\
\hline \multirow{3}{*}{ Boiler low efficiency } & Smoke stack & $\begin{array}{c}\text { High temperature of stack } \\
\text { gas }\end{array}$ & $\begin{array}{l}\text { - Too much excess air } \\
\text { - Dirty firesides }\end{array}$ \\
\hline & Steam pressure & Low steam pressure & $\begin{array}{c}\text { - Low water } \\
\text { - Excessive steam demands } \\
\text { - Poor combustion }\end{array}$ \\
\hline & Combustion & $\begin{array}{l}\text { Combustion gases entering } \\
\text { fire room }\end{array}$ & - Leakage through soot blower casing seal \\
\hline \multirow[t]{2}{*}{$\begin{array}{l}\text { Boiler tubes corro- } \\
\text { sion }\end{array}$} & \multirow[t]{2}{*}{ Fuel } & $\begin{array}{l}\text { Fuel impingement on } \\
\text { furnace walls and tubes }\end{array}$ & $\begin{array}{l}\text { - Incorrect viscosity, temperature, or pressure of } \\
\text { fuel } \\
\text { - Improperly made up atomize assemblies } \\
\text { - water in fuel }\end{array}$ \\
\hline & & High fuel combustion & $\begin{array}{l}\text { - Sudden change in steam demand } \\
\text { - Too much or too little excess air }\end{array}$ \\
\hline
\end{tabular}

Table 4. Pump root cause failure analysis.

\begin{tabular}{|c|c|c|c|}
\hline Failure Mode & Mechanism & Reason & Root Cause \\
\hline $\begin{array}{l}\text { Pump low } \\
\text { efficiency }\end{array}$ & Discharge pressure & Low discharge pressure & - Water excessively hot \\
\hline \multirow{5}{*}{ Pump shutdown } & Impeller & $\begin{array}{c}\text { Low flow rate } \\
\text { \& Low delivery pressure }\end{array}$ & $\begin{array}{c}\text { - Impeller damaged } \\
\text { - Impeller loss on shaft }\end{array}$ \\
\hline & oil & Loss or oil contamination & $\begin{array}{l}\text { - Flooding of oil reservoir } \\
\text { - Over filling of oil reservoir } \\
\text { - Mechanical seal failure } \\
\text { - Improper installation of bearing }\end{array}$ \\
\hline & Low flow & Operation condition & $\begin{array}{c}\text { - Insufficient NPSH } \\
\text { - Water excessively hot } \\
\text { - Impeller damaged or loose on shaft }\end{array}$ \\
\hline & Bearing & High bearing temperature & - Bent shaft \\
\hline & Vibration & $\begin{array}{c}\text { Operation condition } \\
\text { Pump driver motor } \\
\text { Bearing } \\
\text { Mechanical seal }\end{array}$ & $\begin{array}{l}\text { - Operation at low flow } \\
\text { - Misalignment op pump drive motor } \\
\text { - Worm bearing } \\
\text { - Mechanical seal failure }\end{array}$ \\
\hline
\end{tabular}


Table 5. Boiler failure mode effect analysis.

\begin{tabular}{|c|c|c|c|c|c|}
\hline \multirow{2}{*}{ Item } & \multirow{2}{*}{$\begin{array}{l}\text { Failure } \\
\text { Mode }\end{array}$} & \multicolumn{3}{|c|}{ Effect } & \multirow[b]{2}{*}{ LTA } \\
\hline & & Local & System & Plant & \\
\hline \multirow{3}{*}{ Check valve } & Fails to open & Low effect & Low effect & No effect & $\mathrm{Y}$ \\
\hline & Remain open & Low effect & Low effect & No effect & $\mathrm{Y}$ \\
\hline & Crack valve & Low effect & Low effect & No effect & $\mathrm{Y}$ \\
\hline \multirow{6}{*}{$\begin{array}{l}\text { Combustion } \\
\text { room }\end{array}$} & Incorrect burner sequence & Boiler trip & Steam system trip & Production stooping & $\mathrm{Y}$ \\
\hline & Too much fuel being fired & Boiler trip & Steam system trip & Production stooping & $\mathrm{Y}$ \\
\hline & Too much excess air & $\begin{array}{l}\text { Boiler low per- } \\
\text { formance }\end{array}$ & Steam system trip & Lower production & $\mathrm{Y}$ \\
\hline & Faulty flam detector & Boiler trip & Steam system trip & Production stooping & $\mathrm{Y}$ \\
\hline & $\begin{array}{l}\text { Combustion air very Low } \\
\text { flow }\end{array}$ & Boiler trip & Steam system trip & Lower production & $\mathrm{Y}$ \\
\hline & Combustion gas pass failure & Boiler trip & Steam system trip & Lower production & $\mathrm{Y}$ \\
\hline Forced draft fan & Noise in motor & Boiler trip & Steam system trip & Low production & $\mathrm{Y}$ \\
\hline Air filter & Dirt on surface & $\begin{array}{c}\text { Low effect } \\
\text { on boiler }\end{array}$ & Low effect & No effect & $\mathrm{Y}$ \\
\hline Furnace & Hole in tube & $\begin{array}{l}\text { Boiler low effi- } \\
\text { ciency }\end{array}$ & Low output & Low effect & $\mathrm{Y}$ \\
\hline \multirow{2}{*}{ Fuel system } & Relieve valve damage & Boiler trip & Steam system trip & Production stooping & $\mathrm{Y}$ \\
\hline & Faulty of the trip valve & Boiler trip & Steam system trip & Production stooping & $\mathrm{Y}$ \\
\hline Piping system & Corrosion & Boiler shutdown & Steam system trip & Production stooping & $\mathrm{Y}$ \\
\hline Safety valve & Fail to open & Boiler shutdown & Steam system trip & Low productivity & $\mathrm{Y}$ \\
\hline Water softener & Water contamination & Boiler trip & Low output & Low productivity & $\mathrm{Y}$ \\
\hline $\begin{array}{c}\text { Feed water } \\
\text { system }\end{array}$ & Pump trip & Boiler trip & Steam system trip & Production stooping & $\mathrm{Y}$ \\
\hline \multirow{2}{*}{ Water softener } & No softening & $\begin{array}{l}\text { Boiler low effi- } \\
\text { ciency }\end{array}$ & Lower production & No effect & $\mathrm{Y}$ \\
\hline & Too much softening & $\begin{array}{l}\text { Boiler low effi- } \\
\text { ciency }\end{array}$ & Lower production & No effect & $\mathrm{Y}$ \\
\hline
\end{tabular}

Table 6. Pump failure mode effect analysis.

\begin{tabular}{|c|c|c|c|c|c|}
\hline \multirow{2}{*}{ Item } & \multirow{2}{*}{$\begin{array}{c}\text { Failure } \\
\text { Mode }\end{array}$} & \multicolumn{3}{|c|}{ Effect } & \multirow{2}{*}{ LTA } \\
\hline & & Local & Boiler & Steam System & \\
\hline Impeller & Worn impeller & $\begin{array}{c}\text { - Pump low efficiency } \\
\text { - Vibration } \\
\text { - Reduce in suction power }\end{array}$ & Boiler trip & System trip & $\mathrm{Y}$ \\
\hline Bearing & $\begin{array}{l}\text { Faulty thrust } \\
\text { bearing }\end{array}$ & $\begin{array}{l}\text { - Excessive pump vibration } \\
\text { - Motor may be overload } \\
\text { - Increased in shaft } \\
\text { radial movement } \\
\text { - Eventual pump shutdown }\end{array}$ & Boiler shutdown & System shutdown & $\mathrm{Y}$ \\
\hline Shaft & $\begin{array}{l}\text { Shaft deform- } \\
\text { ing }\end{array}$ & $\begin{array}{c}\text { - Pump low efficiency } \\
\text { - Vibration } \\
\text { - Increase in shaft radial movement } \\
\text { - Possible bearing damage } \\
\text { - Eventual coupling failure }\end{array}$ & $\begin{array}{l}\text { - Boiler low } \\
\text { efficiency } \\
\text { - low effect } \\
\text { - low effect } \\
\text { - } \quad \text { low effect }\end{array}$ & $\begin{array}{l}\text { - System low } \\
\text { efficiency } \\
\text { - low effect } \\
\text { - low effect } \\
\text { - low effect }\end{array}$ & $\mathrm{N}$ \\
\hline Casing & Leaking casing & $\begin{array}{l}\text { - Reduce pumping rate } \\
\text { - Possible corrosion on all } \\
\text { pump components }\end{array}$ & $\begin{array}{l}\text { - Boiler low } \\
\text { efficiency } \\
\text { - low effect }\end{array}$ & $\begin{array}{c}\text { - System low effi- } \\
\text { ciency } \\
\text { - low effect }\end{array}$ & $\mathrm{N}$ \\
\hline Coupling & $\begin{array}{l}\text { Faulty shaft } \\
\text { coupling }\end{array}$ & $\begin{array}{l}\text { - Losses of pumping efficiency } \\
\text { - Noise and vibration on the pump } \\
\text { - Possible seals damage } \\
\text { - Eventually pump shutdown }\end{array}$ & $\begin{array}{l}\text { - Boiler low } \\
\text { efficiency } \\
\text { - boiler shutdown } \\
\text { - Boiler trip } \\
\text { - Boiler trip } \\
\end{array}$ & $\begin{array}{l}\text { - System trip } \\
\text { - System trip } \\
\text { - System trip } \\
\text { - System trip }\end{array}$ & Y \\
\hline Ring & $\begin{array}{l}\text { - Faulty impel- } \\
\text { ler wear ring }\end{array}$ & $\begin{array}{l}\text { - Internal liquid leakage } \\
\text { - Eminent impeller wear } \\
\text { - Potential corrosion effect } \\
\text { on pump internals } \\
\text { - Pump capacity greatly } \\
\text { reduce. }\end{array}$ & $\begin{array}{l}\text { - Boiler low } \\
\text { efficiency } \\
\text { - Boiler trip } \\
\text { - Boiler trip } \\
\text { - Boiler low } \\
\text { efficiency }\end{array}$ & $\begin{array}{l}\text { - System low } \\
\text { efficiency } \\
\text { - System trip } \\
\text { - System trip } \\
\text { - System low } \\
\text { efficiency }\end{array}$ & $\mathrm{Y}$ \\
\hline
\end{tabular}


Table 7. Criticality group.

\begin{tabular}{cc}
\hline Group & Criticality Index \\
\hline A & $3-2.5$ \\
B & $2.5-2$ \\
C & $2-1.5$ \\
D & $1.5-1$ \\
\hline
\end{tabular}

Table 8. Criticality analysis for boiler.

\begin{tabular}{|c|c|c|c|c|c|c|c|}
\hline \multirow{2}{*}{ Equipment } & \multirow{2}{*}{$\begin{array}{c}\text { Failure } \\
\text { Mode }\end{array}$} & \multirow{2}{*}{$\begin{array}{c}\text { Failure } \\
\text { Cause }\end{array}$} & \multicolumn{3}{|c|}{ Criticality Analysis } & \multirow{2}{*}{$\begin{array}{c}\text { Criticality } \\
\text { Index }\end{array}$} & \multirow{2}{*}{ Group } \\
\hline & & & Safety & Production & Cost & & \\
\hline \multirow{10}{*}{ Boiler } & \multirow{5}{*}{$\begin{array}{c}\text { Excessive } \\
\text { high steam } \\
\text { outlet tem- } \\
\text { perature }\end{array}$} & Too much excess air & 2 & 1 & 3 & 1.4 & $\mathrm{D}$ \\
\hline & & $\begin{array}{l}\text { Incorrect burner se- } \\
\text { quence }\end{array}$ & 3 & 2 & 1 & 2.2 & B \\
\hline & & $\begin{array}{l}\text { Too much fuel being } \\
\text { fired }\end{array}$ & 3 & 3 & 3 & 3 & A \\
\hline & & $\begin{array}{c}\text { Dirty generating sur- } \\
\text { face }\end{array}$ & 2 & 2 & 3 & 2.2 & B \\
\hline & & Dirty economizer & 3 & 3 & 3 & 3 & $\mathrm{~A}$ \\
\hline & $\begin{array}{l}\text { Low discharge } \\
\text { pressure }\end{array}$ & Water excessively hot & 1 & 3 & 1 & 1.8 & $\mathrm{C}$ \\
\hline & \multirow{2}{*}{$\begin{array}{l}\text { High tem- } \\
\text { perature of } \\
\text { stack gas }\end{array}$} & Too much excess air & 2 & 1 & 3 & 1.8 & $\mathrm{C}$ \\
\hline & & Dirty firesides & 3 & 2 & 3 & 2.6 & A \\
\hline & \multirow{2}{*}{$\begin{array}{l}\text { Combustion } \\
\text { gases entering } \\
\text { fire room }\end{array}$} & $\begin{array}{l}\text { Leakage through soot } \\
\text { blower casing seal }\end{array}$ & 3 & 3 & 3 & 3 & A \\
\hline & & $\begin{array}{l}\text { Leakage through } \\
\text { economizer drain line }\end{array}$ & 3 & 3 & 3 & 3 & A \\
\hline
\end{tabular}

Table 9. Criticality analysis for the pump.

\begin{tabular}{|c|c|c|c|c|c|c|c|}
\hline \multirow{2}{*}{ Equipment } & \multirow{2}{*}{$\begin{array}{l}\text { Failure } \\
\text { Mode }\end{array}$} & \multirow{2}{*}{$\begin{array}{l}\text { Failure } \\
\text { Cause }\end{array}$} & \multicolumn{3}{|c|}{ Criticality Analysis } & \multirow{2}{*}{$\begin{array}{l}\text { Criticality } \\
\text { Index }\end{array}$} & \multirow{2}{*}{ Group } \\
\hline & & & Safety & Production & Cost & & \\
\hline \multirow{8}{*}{ Pump } & $\begin{array}{c}\text { Low discharge } \\
\text { pressure }\end{array}$ & $\begin{array}{l}\text { Water excessively } \\
\text { hot }\end{array}$ & 2 & 3 & 1 & 2.2 & B \\
\hline & \multirow{4}{*}{$\begin{array}{l}\text { High bearing } \\
\text { temperature }\end{array}$} & Bent shaft & 3 & 3 & 3 & 3 & $\mathrm{~A}$ \\
\hline & & Worm bearing & 3 & 3 & 2 & 2.8 & A \\
\hline & & Lack of lubrication & 3 & 3 & 2 & 2.8 & A \\
\hline & & $\begin{array}{l}\text { Improper installa- } \\
\text { tion of bearing }\end{array}$ & 3 & 3 & 2 & 2.8 & A \\
\hline & \multirow{2}{*}{$\begin{array}{l}\text { Pump casing } \\
\text { overheats }\end{array}$} & $\begin{array}{l}\text { Misalignment of pump } \\
\text { drive motor }\end{array}$ & 3 & 3 & 3 & 3 & A \\
\hline & & Shaft sleeve worn & 3 & 3 & 3 & 3 & A \\
\hline & Low flow & $\begin{array}{l}\text { Impeller damaged } \\
\text { or loose on shaft }\end{array}$ & 3 & 3 & 3 & 3 & A \\
\hline
\end{tabular}

(if any) of the proactive tasks is technically feasible in any context, and if so for deciding how often they should be done and who should do them.

Whether or not a proactive task is technically feasible is governed by the technical characteristics of the task and of the failure which it is meant to prevent. Whether it is worth doing is governed by how well it deals with the consequences of the failure. If a proactive task cannot be found which is both technically feasible and worth doing, then suitable default action must be taken. Maintenance tasks are consisting of run-to-failure (RTF), time-directed maintenance, condition-directed maintenance (CD) and failure-finding (FF). The maintenance task for the boiler is illustrated in Table $\mathbf{1 0 .}$

\subsection{Maintenance Labor Force}

The maintenance labor force is presented in Table 11. This table shows the size of maintenance labor force calculations for the PM levels (six monthly, monthly and weekly). In addition, the labor saving cost is introduced in Table 12. Not that the proposed labor cost (295200 \$/year) decreased with respect to the current values (220800 \$/year). 
Table 10. Maintenance task.

\begin{tabular}{|c|c|c|c|c|c|c|}
\hline Equipment & $\begin{array}{l}\text { Failure } \\
\text { Mode }\end{array}$ & $\begin{array}{l}\text { Failure } \\
\text { Cause }\end{array}$ & Group & Task & Description & Frequency \\
\hline \multirow{7}{*}{ Boiler } & \multirow{7}{*}{$\begin{array}{l}\text { Excessive high } \\
\text { steam outlet } \\
\text { temperature }\end{array}$} & $\begin{array}{l}\text { Too much excess } \\
\text { air }\end{array}$ & $\mathrm{D}$ & RTF & n........... & (n......... \\
\hline & & \multirow{3}{*}{$\begin{array}{l}\text { Incorrect burner } \\
\text { sequence }\end{array}$} & \multirow{3}{*}{ B } & CD & $\begin{array}{l}\text { Measure the diameter of } \\
\text { the fuel opening holes } \\
\text { inside burner }\end{array}$ & M \\
\hline & & & & TD & $\begin{array}{l}\text { Replacement of fuel hose } \\
\text { and gaskets }\end{array}$ & $\mathrm{S}$ \\
\hline & & & & $\mathrm{FF}$ & $\begin{array}{c}\text { Check the deflector } \\
\text { position }\end{array}$ & W \\
\hline & & $\begin{array}{l}\text { Too much fuel } \\
\text { being fired }\end{array}$ & A & CD & $\begin{array}{l}\text { Measure the diameter of } \\
\text { the fuel opening holes } \\
\text { inside burner }\end{array}$ & M \\
\hline & & $\begin{array}{l}\text { Dirty generating } \\
\text { surface }\end{array}$ & B & $\mathrm{CD}$ & $\begin{array}{l}\text { Cleaning generating } \\
\text { surface }\end{array}$ & $\mathrm{S}$ \\
\hline & & Dirty economizer & A & $\mathrm{CD}$ & $\begin{array}{l}\text { Measure the temperature } \\
\text { of the stack gases }\end{array}$ & M \\
\hline
\end{tabular}

Table 11. The size of maintenance labor force.

\begin{tabular}{ccccc}
\hline PM Level & Frequency & $\begin{array}{c}\text { Duration } \\
\text { (Hours) }\end{array}$ & No. of Workers & Man-hour per PM level \\
\hline Six Monthly & 2 & 21 & 4 & 168 \\
Monthly & 10 & 5 & 2 & 100 \\
Weakly & 50 & 6.15 & 1 & 325 \\
\hline
\end{tabular}

Maintenance labor force $=1$ labor.

Table 12. Labor saving cost.

\begin{tabular}{|c|c|c|c|}
\hline Item & $\begin{array}{c}\text { Labor } \\
\text { type }\end{array}$ & $\begin{array}{c}\text { Number of labors } \\
\text { Per day } \\
\text { (current maintenance) }\end{array}$ & $\begin{array}{c}\text { Number of labors } \\
\text { Per day } \\
\text { (proposed ) }\end{array}$ \\
\hline \multirow{3}{*}{$\begin{array}{c}\text { Engineers } \\
\text { (1000\$/month) }\end{array}$} & Mechanical & 5 & 4 \\
\hline & Electrical & 5 & 4 \\
\hline & Control & 5 & 4 \\
\hline \multirow{2}{*}{$\begin{array}{l}\text { Technicians } \\
\text { (800\$/month) }\end{array}$} & Mechanical & 6 & 4 \\
\hline & Electrical & 6 & 4 \\
\hline $\begin{array}{l}\text { Total cost } \\
\text { (\$)/year) }\end{array}$ & & 295200 & 220800 \\
\hline \multicolumn{4}{|c|}{ Saving cost $(\%)=25.2$} \\
\hline
\end{tabular}

\section{Downtime Cost}

- Average CM downtime of the feed water pump = $20 \mathrm{hr} /$ year.

- Average CM downtime of the fire tube boiler = $30 \mathrm{hr} /$ year.

- Average CM downtime of the system auxiliaries = $10 \mathrm{hr} /$ year.

- Average down cost rate $=10000 \$ / \mathrm{hr}$

- Total downtime cost rate $=600,000 \$ /$ year

- Proposed saving downtime cost $=480,000$ \$/year

Spare Parts Program

The proposed spare parts program is shown in Table 13. This table shows that the spare parts for the plant main components (feed-water pump, water tube boiler ant steam turbine). Proposed spare parts program results indicate a saving of about $22.17 \%$ of the spare parts total cost as compared with that of the current maintenance (CM).

\section{Conclusions}

The results of the RCM technique applied of the plant show that the PM proposed tasks and planning are generated. Moreover, PM is consisted of on-condition and scheduled maintenance. The RCM had great impact on the PM tasks. The Run-To-Failure (RTF) frequency has been decreased. It is recommended to perform these tasks (CD, TD and FF) every yearly, six monthly and monthly. 
Table 13. Proposed spare parts program (yearly).

\begin{tabular}{|c|c|c|c|c|c|}
\hline Equipment & Spare part & $\begin{array}{l}\text { Quantity } \\
\text { (Current) }\end{array}$ & $\begin{array}{c}\text { Cost (current) } \\
\$ / \text { year }\end{array}$ & $\begin{array}{c}\text { Quantity } \\
\text { (Proposed) }\end{array}$ & $\begin{array}{c}\text { Cost (Proposed) } \\
\$ / \text { year }\end{array}$ \\
\hline \multirow{4}{*}{$\begin{array}{c}\text { Feed } \\
\text { water pump }\end{array}$} & Coupling & 2 & 3000 & 1 & 1500 \\
\hline & Mechanical seal & 6 & 12000 & 4 & 8000 \\
\hline & Motor bearing & 6 & 24000 & 4 & 16000 \\
\hline & Pump bearing & 6 & 12000 & 4 & 8000 \\
\hline \multirow{3}{*}{ Fire tube boiler } & Gasket & 12 & 12000 & 10 & 10000 \\
\hline & Fuel house & 2 & 40000 & 2 & 40000 \\
\hline & Fan bearing & 4 & 12000 & 2 & 6000 \\
\hline $\begin{array}{c}\text { Total cost } \\
\text { (\$/year) }\end{array}$ & & & 115000 & & 89500 \\
\hline Saving cost \% & & & & 22.17 & \\
\hline
\end{tabular}

The proposed labor program is carried out. The results show that the labor cost decreases from 295200 \$/year to 220800 \$/year (about 25.2\% of the total labor cost) for the proposed PM planning. Moreover, the downtime cost (DTC) of the co-generation plant components is investigated. The proposed PM planning results indicate a saving of about $80 \%$ of the total downtime cost as compared with that of current maintenance (RTF). The system reliability increase with decreasing the labor cost.

The proposed spare parts program for the co-generation plant components (feed water pump, boiler and turbo-generator) are generated. The results show that about $22.17 \%$ of the annual spare parts cost are saved when proposed PM planning other current maintenance (RTF) once.

\section{Acknowledgements}

The author wishes to express his thanks to the staff members of the Egyptian Salts \& Minerals Company (Emisal), El-Fayom, Egypt, for their support during carrying out this work.

\section{References}

[1] M. Dixey, "Putting Reliability at the Center of Maintenance," Professional Engineering, Vol. 6, No. 6, June 1993, pp. 23-25.
[2] S. A. Abdulrohim, O. D. Salih and A. Raouf, "RCM Concepts and Application: A Case Study," International Journal of Industrial Engineering, Vol. 7, No. 2, 2000, pp. 123-132.

[3] J. Wang and J. Chu, "Selection of Optimum Maintenance Strategies Based on a Fuzzy Analytic Hierarchy Process," International Journal of Production Economics, Vol. 107, No. 1, 2007, pp. 151-163.

[4] R. K. Sharma, D. Kumar and P. Kumar, "FLM to Select Suitable Maintenance Strategy in Process Industries Using MISO Model,” Journal of Quality in Maintenance Engineering, Vol. 11, No. 4, 2005, pp. 359-374.

[5] The National Aeronautics and Space Administration, "Reliability-Centered Maintenance Guide for Facilities and Collateral Equipment," NASA, Washington D.C., February 2000.

[6] A. M. Smith, "Reliability-Centered Maintenance,” McGrawHill, New York, 1993.

[7] J. Shayeri, "Development of Computer-Aided Maintenance Resources Planning (CAMRP): A Case of Multiple CNC Machining Centers," Robotics and ComputerIntegrated Manufacturing, Vol. 23, No. 6, 2007, pp. 614623.

[8] M. Rausand, "Reliability-Centered Maintenance,” Reliability Engineering and System Safety, Vol. 60, No. 2, 1998, pp. 121-132.

[9] A. H. Gomaa, "Maintenance Planning and Management," A Literature Study, American University in Cairo, Cairo, 2003. 